\title{
Médecine de l'obésité et pneumologie : il faut encore et encore développer des collaborations, pour une prise en charge optimale des apnées-hypopnées obstructives du sommeil et du syndrome obésité-hypoventilation !
}

\author{
O. Ziegler \\ (C) Lavoisier SAS 2015
}

Ce numéro thématique, particulièrement riche, nous propose des analyses, des synthèses ou des débats pertinents sur des comorbidités de l'obésité, à la fois fréquentes et graves, le syndrome des apnées-hypopnées obstructives du sommeil (SAHOS) et le syndrome obésité-hypoventilation ( $\mathrm{SOH})$. La Haute Autorité de Santé vient de publier de nouvelles recommandations dans ce domaine (HAS 10 septembre 2014), ce qui montre bien l'intérêt du sujet !

Si le SAHOS est depuis des années l'objet de toutes les attentions, du pneumologue au cardiologue en passant par le diabétologue et le médecin nutritionniste, le $\mathrm{SOH}$ a été longtemps quelque peu ignoré, en dehors du cercle des spécialistes. Certes la CIM 10 (classification internationale des maladies) en avait fait l'un des rares codes (E 66.2 : «Obésité extrême avec hypoventilation alvéolaire ») attribués à l'obésité. Mais, en pratique, il faut bien reconnaître que la prise en charge de cette redoutable complication est loin d'être optimale.

Et pourtant l'hypoxie, qu'elle soit intermittente comme dans le SAHOS ou permanente comme dans le SOH apparaît comme un puissant marqueur de risque à différents niveaux.

Bien des complications métaboliques de l'obésité sont la conséquence d'un dysfonctionnement du tissu adipeux. Celui-ci ne joue plus son rôle physiologique : il ne stocke plus les acides gras (AG) en période post prandiale (lipogenèse insuffisante) d'une part, et libère un excès d'AG libres à distance des repas (lipolyse excessive) d'autre part. Cette mauvaise gestion des flux d'AG a de multiples conséquences systémiques : insulino-résistance, inflammation de bas grade, dépôts ectopiques de lipides, lipotoxicité... L'hypertrophie des adipocytes est la première étape d'une longue série de réactions en chaîne, qui conduit à l'insulino-

\section{O. Ziegler $(\bowtie)$}

Service de Diabétologie, Maladies Métaboliques, Nutrition, CHRU de Nancy - Hôpital Brabois Adulte, rue du Morvan, 54500 Vandoeuvre-les-Nancy

e-mail : o.ziegler@chu-nancy.fr résistance et au remodelage fibro-inflammatoire de ce tissu adipeux qui devient pathologique... L'hypoxie locale, qu'elle résulte d'altérations fonctionnelles de la microcirculation ou d'une angiogénèse insuffisante, pourrait jouer un rôle physiopathologique, qui reste néanmoins débattu.

De nombreux travaux suggèrent que l'hypoxie intermittente du SAOS est un facteur aggravant de l'insulinorésistance et un facteur de risque des troubles de la glycorégulation qui peuvent mener au diabète de type 2 . Il est hautement probable qu'elle puisse majorer aussi la dysfonction du tissu adipeux. La situation d'hypoxie-hypercapnie du $\mathrm{SOH}$ correspondrait alors au niveau supérieur de gravité.

\section{Ce que les « médecins de l'obésité » doivent savoir}

\section{A propos du SAHOS}

Le dépistage du SAHOS devrait être « obligatoire »! Il est cependant nécessaire de le cibler sur la population la plus à risque, compte tenu du coût économique de la polygraphie ou de la polysomnographie, qui sont indispensables ! Le lecteur découvrira le STOP-BANG, un acronyme utile pour analyser la présomption clinique, tout en se rappelant que les arguments cliniques ne suffisent pas !

Il nous paraît raisonnable de faire ce dépistage quand l'obésité est sévère, de classe 2 (IMC $\geq 35 \mathrm{~kg} / \mathrm{m}^{2}$ ) ou quand elle est franchement abdominale et associée à un syndrome métabolique, même si l'IMC est inférieur à ce seuil arbitraire.

Le clinicien ne peut plus ignorer que le SAHOS est actuellement considéré non seulement comme un facteur de risque cardiovasculaire (maladie coronaire et pathologie cérébro-vasculaire), mais aussi comme un facteur aggravant d'une maladie cardio-vasculaire existante (par exemple : effet défavorable sur la mortalité coronaire). En traitant le SAHOS par pression positive continue (PPC), il semble 
possible d'améliorer le pronostic cardio-vasculaire, sous réserve d'une bonne observance.

Le SAOS est aussi un problème majeur chez l'enfant obèse. Sa prévalence semble aussi élevée que chez l'adulte, les études épidémiologiques restant limitées à de petites séries. Le facteur « durée de l'exposition » étant un élément clef pour le développement des pathologies chroniques, on peut être inquiet quant aux conséquences cardio-métaboliques des hypoxies intermittentes qui débutent dans l'enfance. De plus, le SAHOS peut être à l'origine de difficultés scolaires et d'une baisse de l'estime de soi, qui compromettent les apprentissages et le développement cognitif des enfants ou adolescents concernés.

Si le principe du traitement est le même, le clinicien ne doit pas oublier de chercher une hypertrophie des tissus lymphoïdes des voies aériennes supérieures. L'adénoïdectomie et/ou l'amygdalectomie peuvent être utiles, même si cette chirurgie ORL est moins efficace chez l'enfant obèse, du fait de l'infiltration des tissus mous du pharynx par le tissu adipeux.

\section{Prise en charge périopératoire du SAHOS chez le sujet obèse}

Le SAHOS devrait être dépisté et traité avant toute intervention chirurgicale, car les risques de complications périopératoires sont élevés. L'intubation et la ventilation peuvent être difficiles pour des raisons anatomiques. Le risque de détresse respiratoire post-extubation, secondaire à la formation d'atélectasies ou à l'obstruction des voies aériennes n'est pas négligeable ; il est parfois associé à l'utilisation de dérivés morphiniques.

De nombreuses précautions doivent être envisagées : utilisation d'une PPC pré, per et postopératoire, mise en place de protocoles de ventilation au masque et/ou d' « intubation difficile », recours à la ventilation protectrice, épargne morphinique, positionnement semi-assis, avec un monitorage rapproché en postopératoire...

\section{A propos du SOH}

Le syndrome obésité-hypoventilation ( $\mathrm{SOH}$ ) ou «syndrome de Pickwick» est considéré comme une des principales causes d'insuffisance respiratoire chronique, après la bronchopneumopathie chronique obstructive (BPCO). Sa prévalence augmente avec la sévérité de l'obésité : 10 à $20 \%$, voire davantage en cas d'obésité massive (IMC $\geq 40 \mathrm{~kg} / \mathrm{m}^{2}$ ). Les données, peu nombreuses il est vrai, sur la mortalité sont inquiétantes.

Le diagnostic est souvent tardif à l'occasion d'un épisode d'insuffisance respiratoire aigue, qui impose une hospitalisation en urgence. Il ne faut pas compter sur les signes cliniques qui sont minimisés par le patient : la dyspnée d'effort est souvent masquée par le handicap lié à l'obésité sévère ou par une gonarthrose par exemple, qui limite les mouvements. La fatigue, la somnolence, les céphalées sont peu spécifiques. Les signes d'insuffisance ventriculaire droite dans les formes compliquées de cœur pulmonaire chronique ne sont pas si faciles à détecter dans ce contexte ...

Le diagnostic repose sur une mesure des gaz du sang artériel : $\mathrm{PaCO}_{2}$ diurne $\geq 45 \mathrm{mmHg}$, l'hypoxémie $\left(\mathrm{PaO}_{2}<70\right.$ $\mathrm{mmHg}$ ) n'étant pas requise, mais souvent présente. Il faut penser à doser les bicarbonates car une élévation chronique de leur taux sérique ( $\geq 27 \mathrm{mEq} / \mathrm{l}$ ) est évocatrice d'une alcalose métabolique. Enfin, chercher un $\mathrm{SOH}$ en présence d'un SAHOS (et vice et versa) est nécessaire, car l'association est fréquente.

La prise en charge spécialisée est indispensable compte tenu de la gravité du pronostic. Elle comporte l'étude du retentissement cardiaque et la rechercher d'une d'hypertension pulmonaire.

\section{Ce que les pneumologues doivent connaître de l'obésité sévère et de son traitement}

Le traitement de l'obésité sévère est rarement facile. Les injonctions, les bons conseils sont inopérants et contribuent à ce sentiment de stigmatisation dont souffrent les personnes obèses.

Le potentiel de perte de poids, pour reprendre une expression d'Arnaud Basdevant n'est pas facile à déterminer au niveau individuel. Il dépend de multiples facteurs biologiques, comportementaux et psycho-sociaux. On sait maintenant que la fibrose du tissu adipeux et la sarcopénie, par exemple, sont des éléments péjoratifs.

Les Modifications Thérapeutiques du Mode de Vie (MTMV) sont la clef du traitement, car la maladie a une forte composante comportementale (Ziegler 2014). Leur mise en œuvre implique que la personne obèse sorte d'un état de « résignation apprise » qui s'installe au fil des échecs pour retrouver un sentiment d'efficacité. L'intervention d'une équipe pluri-professionnelle dans le cadre d'un parcours d'éducation thérapeutique est un progrès utile (Ziegler 2014).

Il faut hélas envisager une perte de poids importante pour améliorer la fonction respiratoire, difficile à obtenir et surtout à maintenir sur le long terme. Les nouveaux médicaments de l'obésité pourraient représenter un certain progrès, les bons répondeurs pouvant obtenir une perte de poids de $15 \%$, mais leurs effets secondaires suscitent débats et controverses. Ils ne sont pas disponibles en France.

La chirurgie bariatrique est une excellente solution à condition de bien évaluer la balance bénéfice-risque d'une part et la motivation réelle du patient d'autre part. Ce terme impropre désigne à la fois le désir du patient et son aptitude à s'adapter aux conséquences digestives de cette chirurgie. 
Tableau 1 Quelques facteurs associés à la mortalité à 30 jours après court circuit gastrique (effet non ajusté sur les autres comorbidités). Les Facteurs de risque indépendants dans l'analyse multivariée sont l'IMC, l'âge, le sexe masculin, l'hypertension pulmonaire, l'insuffisance cardiaque congestive et les maladies hépatiques (Benotti et al, 2014).

\begin{tabular}{|llllc|}
\hline Paramètres (liste non exhaustive) & Prévalence \% & Mortalité \% si présent & Mortalité \% si absent & p \\
\hline Hypertension artérielle & $52 \%$ & $0,21 \%$ & $0,09 \%$ & $<0,0001$ \\
Insuffisance cardiaque congestive & $2,4 \%$ & $0,83 \%$ & $0,13 \%$ & 0,0001 \\
Maladie coronaire & $3 \%$ & $0,45 \%$ & $0,14 \%$ & 0,0001 \\
Artériopathie périphérique & $1,2 \%$ & $0,50 \%$ & $0,15 \%$ & $<0044$ \\
Diabète & $31,5 \%$ & $0,24 \%$ & $0,11 \%$ & 0,0001 \\
SAS & $36,4 \%$ & $0,18 \%$ & $0,13 \%$ & 0,056 \\
Obésité- hypoventilation & $2 \%$ & $0,49 \%$ & $0,14 \%$ & $<0,0001$ \\
Hypertension pulmonaire & $0,5 \%$ & $1,59 \%$ & $0,14 \%$ & $<0,0001$ \\
Handicap fonctionnel majeur & $3,6 \%$ & $0,44 \%$ & $0,14 \%$ & $<$ \\
\hline
\end{tabular}

Le problème est donc d'évaluer les risques en cas de pathologie respiratoire. Le SAHOS traité n'est probablement plus un facteur de morbi-mortalité opératoire. Mais le $\mathrm{SOH}$ et surtout l'hypertension pulmonaire le sont, comme le montrent les données d'un registre américain, récemment publiées (Tableau 1) concernant la mortalité à 30 jours du court circuit gastrique, qui est ici de $0,15 \%$. On remarquera d'abord que la prévalence du SAHOS est probablement sous-estimée dans cette étude (36,4\%). Bien peu de patients présentant un $\mathrm{SOH}$ ou une hypertension pulmonaire sont opérés, dans la vraie vie; de fait le nombre de décès est respectivement multiplié par 3 et par 11 en présence de ces co-morbidités.

\section{Conclusion}

L'association du SAHOS avec les différentes composantes du syndrome métabolique ou avec ses complications (diabète de type 2 , maladie cardio-vasculaire) est si fréquente qu'on aurait envie d'en faire la manifestation respiratoire de ce syndrome. Les hépatologues ont montré l'exemple avec la stéato-hépatite métabolique (NASH). On peut d'ailleurs remarquer que le SAHOS et la NASH sont devenus des facteurs de risque cardio-vasculaires pour de nombreux experts ! Néanmoins, la part attribuable à ces nouveaux acteurs dans l'augmentation du risque n'est pas facile à établir.

Si des liens de causalité ne sont pas encore totalement établis, l'intérêt pédagogique pour le clinicien est évident. Il faut chercher un SAHOS chez les patients obèses « métaboliques », a fortiori s'ils sont diabétiques, comme il faut le faire pour la NASH. Le SOH doit être considérée comme une véritable insuffisance respiratoire du sujet obèse, dont le pronostic est défavorable à moyen terme.

On ne peut malheureusement se soustraire à l'analyse économique car les traitements de suppléance par appareil- lage respiratoire ont un coût considérable. En France près de 530000 patients ont été traités par PPC en 2013 et les chiffres augmentent chaque année ! L'appareil à PPC est loué à un prestataire pour un coût moyen d'environ $20 €$ par semaine, pris en charge à $60 \%$ par l'assurance maladie, le reste l'étant par les assurances complémentaires (Nau 2014). En 2012, pour le régime général, le montant total remboursé pour les PPC était de $298965358 €$ (HAS 2014). L'arrêté du 22 octobre 2013 du ministère de la santé définit de nouvelles modalités de prise en charge des appareils à PPC : utilisation de l'appareil durant un temps minimum par nuit et obligation pour le prestataire de mettre en place un système de « téléobservance ». Ces mesures suscitent évidemment bien des réactions (Nau 2014). On peut en effet considérer que dans $20 \%$ des cas les patient n'utilisent pas ou peu le matériel de PPC mis à leur disposition, ce qui représenterait une « dépense non justifiée » de 80 millions d'euros par an.

On voit ici l'intérêt de l'approche éducative centrée sur le point de vue du patient et sur sa motivation. On réalise aussi qu'il faut investir dans la prévention et s'appuyer sur les centres spécialisés de l'obésité (CSO) mis en place dans toutes les régions, pour prendre en charge les cas difficiles.

\section{Bibliographie}

Baguet JP (2015) Apnées obstructives du sommeil et complications cardiovasculaires. Obésité 10 : à venir

Weitzenblum E, Kessler R, Chaouat A, Canuet M (2015) Le syndrome d'hypoventilation alvéolaire dans l'obésité : le «syndrome obésité-hypoventilation ». Obésité 10 : à venir

Böhme P, Corbonnois P, Duchesne L, Quilliot D, Ziegler O. (2015) Adiposité, hypoxie et apnées du sommeil : de l'obésité au syndrome métabolique. Obésité 10 : à venir

Aubertin G, Dubern B (2015) Particularités du syndrome d'apnées obstructives du sommeil de l'enfant et de l'adolescent obèses. Obésité 10 : à venir 
Shoukri A, Dubé BP, Bermejo JG, Laveneziana P (2015) Les contraintes mécaniques respiratoires au repos et à l'effort du sujet obèse. Obésité 10 : à venir

De Jong A, Monnin M, Trinh Duc P, Cahnques G, Futier E, Jaber S (2015) Prise en charge périopératoire du syndrome d'apnées du sommeil chez le sujet obèse. Obésité 10 : à venir

Flávia Gabe B, Nguyen-Plantin XL, Fleury B (2015) Syndrome d'Apnées Obstructives du Sommeil et Obésité : quels traitements ? Obésité 10 : à venir

Benotti P, Wood GC, Winegar DA, et al (2014) Risk Factors Associated With Mortality After Roux-en-Y Gastric Bypass Surgery. Ann Surg 259: 123-30

HAS 2014 : Évaluation clinique et économique des dispositifs médicaux et prestations associées pour la prise en charge du syndrome d'apnées-hypopnées obstructives du sommeil (SAHOS) », validé par la Commission nationale d'évaluation des dispositifs et technologies de santé de la HAS le 15 juillet 2014. http://www.has-sante.fr/portail/ jcms/c_1761160/fr/apnees-du-sommeil-de-nouvelles-recommandationsde-prise-en-charge-des-patients

HAS 2014 Volet 2 : Évaluation économique et analyse des bases de données. http://www.has-sante.fr/portail/upload/docs/application/pdf/ 2014-09/rapport_sahos_-_evaluation_economique.pdf

Nau JY (2014). Apnéique, attention : si tu ne suis pas ton traitement, ton air sera payant ttp://jeanyvesnau.com/2014/01/14/apneiqueattention-si-tu-ne-suis-pas-ton-traitement-ton-air-sera-payant/

Ziegler O (2014) Éducation thérapeutique et parcours de soins de la personne obèse. Synthèse et propositions. Obésité $9: 225-33$

Ziegler O, Bertin E, Jouret B, Calvar R, Sanguignol F, Avignon A, Basdevant A. (2014) Éducation thérapeutique et parcours de soins de la personne obèse. Référentiel et organisation. Rapport à la Direction Générale de la Santé et à la Direction Générale de l'Offre de Soins. Obésité 9:302-28 